\title{
DISTURBANCES IN A SEMI-INFINITE PIEZOELECTRIC MEDIUM COATED WITH A PERFECTLY CONDUCTING THIN FILM UNDER THE INFLUENCE OF TIME-DEPENDENT FLOW OF HEAT FOR A FINITE INTERVAL
}

\author{
T.K. MUNSHI
}

Department of Physics, Kharagpur College

P.O. Kharagpur, Dist. Midnapore, West Bengal, India

\section{K.K. KundU}

Department of Physics, City College, Calcutta 700009, India

\author{
and R.K. Mahalanabis \\ Department of Mathematics, Jadavpur University, Calcutta 700032, India
}

(Received April 23, 1993)

An attempt was made to investigate analytically the mechanical behaviour of a semi-infinite piezoelectric medium coated with a perfectly conducting thin film under the influence of time-dependent flow of heat at the finite end. The problem involves the interaction of three fields, viz. electrical, mechanical and thermal, and the solution was obtained with the aid of operational calculus. The resultant displacement consists of a time-dependent, as well as a time-independent part and the variation of displacements exhibits an arc of a parabola.

PACS numbers: 77.70.+a

\section{Introduction}

The investigation of the disturbances of a piezoelectric material from the standpoint of mechanics of continuous media have been initiated by Haskins and Walsh [1], Mc Quarrie and Buessem [2], Cady [3], Mindlin [4], Redwood [5] etc. The relevant problems are extremely important in view of their applications in the fields of ultrasonics and acoustic engineering. Researchers like Redwood [5], Kaliski [6], Balabaev and Ivina [7], Karlash [8], Chen [9] considered problems on piezoelectricity without taking into consideration the effect of thermal field but the 
problems of thermopiezoelectricity, i.e. pyroelectricity, have not been considered extensively. Experimental researches reveal that a perfectly conducting thin film on a piezoelectric solid influences the nature of propagation in the material. The present study attempts to investigate analytically the nature of mechanical disturbances produced in a piezoelectric material coated with a perfectly conducting thin film when the finite end is subjected to a

a) temperature varying exponentially with time and acting for a finite interval.

b) temperature varying periodically with time and acting for a finite interval.

This constitutes an interesting problem of the interaction of three different fields, viz. electrical, mechanical and thermal, and finally the mechanical displacement produced in each case has been obtained by applying operational calculus. The mechanical displacement consists of two parts: a time-dependent and a time-independent part. For time-dependent part Figs. 2 and 3 represent arcs of parabola in both cases.

\section{The problem and boundary conditions}

A semi-infinite piezoelectric medium is considered and the origin of the axes of coordinates is chosen at the finite end of the surface of the medium. The $x$-axis is drawn normally into the medium and $y-z$ plane is the surface. This free surface is then coated with a perfectly conducting thin film, so that the electric fields on the plane surface are short-circuited (Fig. 1). This means that

$$
E_{y}=0 \text { on } x=0 \text {. }
$$

If $\theta(x, t)$ is the excess of temperature over $\theta_{0}$, the temperature of the piezoelectric medium in a state of zero stress and strain, we can assume $\theta$ to be small so that the elastic and thermal properties of the material remain undisturbed throughout the time under consideration. If $\theta(x, t)$ is the temperature field and $T$ - the mechanical stress applied on the free surface $x=0$, we assume the following boundary and regularity conditions:

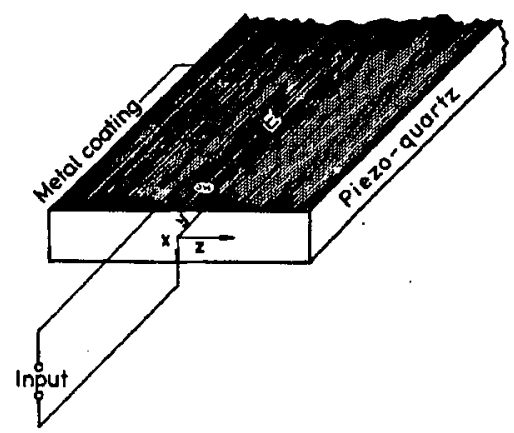

Fig. 1. Schematic illustration of the conducting thin-film-coated piezoquartz and coordinate axes. 
Case 1

$$
\begin{aligned}
& \theta= \begin{cases}\theta_{0} \mathrm{e}^{-\omega t}, & 0 \leq t \leq \tau, \\
0, & t>\tau,\end{cases} \\
& T(0, t)=T_{0} \delta(t), \\
& \theta(x, t), \quad u(x, t) \rightarrow 0 \text { as } x \rightarrow \infty .
\end{aligned}
$$

\section{Case 2}

$$
\begin{aligned}
& \theta= \begin{cases}\theta_{0} \sin \omega t, & 0 \leq t \leq \tau, \\
0, & t>\tau,\end{cases} \\
& T(0, t)=T_{0} \mathrm{e}^{\omega t}[H(t)-H(t-\tau)], \\
& \theta(x, t), \quad u(x, t) \rightarrow 0 \text { as } x \rightarrow \infty,
\end{aligned}
$$

where $u$ is the mechanical displacement in the $x$-direction, $\theta_{0}, T_{0}$ and $\omega$ are constants, $\delta(t)$ is the Dirac delta function and $H(t)$ is the Heaviside unit function. The basic equations of the problem are

$$
\begin{aligned}
& \frac{\partial^{2} u}{\partial x^{2}}-\frac{\rho}{c} \frac{\partial^{2} u}{\partial t^{2}}=-\frac{e}{c} \frac{\partial E}{\partial x}+k_{1} \theta, \\
& \frac{\partial^{2} E}{\partial x^{2}}-\mu \varepsilon \frac{\partial^{2} E}{\partial t^{2}}=-\mu e \frac{\partial^{2}}{\partial t^{2}}\left(\frac{\partial u}{\partial x}\right)+k_{2} \theta,
\end{aligned}
$$

where $E$ is the electric field in the $y$-direction, $\rho$ is the density of the material, $c, \mu$ are the elastic constants, $e$ - the piezoelectric constant, $\varepsilon$ - dielectric permittivity, $k_{1}, k_{2}$ are the thermopiezoelectric constants. The other fundamental equation is the heat conduction equation

$$
k \frac{\partial^{2} \theta}{\partial x^{2}}=\frac{\partial \theta}{\partial t}
$$

where $k$ is the thermal diffusivity constant.

\section{Solution of the problem}

To solve the problem we make use of the Laplace transform of parameter $p$. Now taking the transform of Eqs. (4) to (6) and the boundary condition (1) to (3) we obtain

$$
\begin{aligned}
& \left(D^{2}-\frac{\rho}{c} p^{2}\right) \bar{u}+\frac{e}{c} D \bar{E}=k_{1} \bar{\theta} \\
& \mu e p^{2} D \bar{u}+\left(D^{2}-\mu \varepsilon p^{2}\right) \bar{E}=k_{2} \bar{\theta} \\
& \left(D^{2}-p / k\right) \bar{\theta}=0 .
\end{aligned}
$$

Now eliminating $\bar{E}$ from Eqs. (7) and (8), we get

$$
\left[D^{4}-\left(\frac{\rho}{c}+\mu \varepsilon+\frac{\mu e^{2}}{c}\right) p^{2} D^{2}+\frac{\mu \varepsilon \rho}{c} p^{4}\right] \bar{u}=\left(k_{1} D^{2}-\frac{e}{c} k_{2} L-\mu \varepsilon p^{2} k_{1}\right) \bar{\theta} .
$$

For case 1

$$
\bar{E}_{y}=0
$$




$$
\begin{aligned}
& \bar{\theta}=\frac{\theta_{0}}{p+\omega}\left[1-\mathrm{e}^{-(p+\omega) \tau}\right], \\
& \bar{T}=T_{0}, \\
& \bar{\theta}(x, p), \bar{u}(x, p) \rightarrow 0 \text { as } x \rightarrow \infty .
\end{aligned}
$$

Now solving Eq. (9) satisfying the regularity and boundary condition of Eq. (11), we get

$$
\vec{\theta}(x, p)=\frac{\theta_{0}}{p+\omega} \mathrm{e}^{-\sqrt{p / k} x}\left[1-\mathrm{e}^{-(p+\omega) \tau}\right] .
$$

Substituting the expression for $\bar{\theta}(x, p)$ from Eq. (12) into Eq. (10), we get

$$
\left(D^{4}-A p^{2} D^{2}+B p^{4}\right) \bar{u}=x_{1}(p) x_{2}(p) \mathrm{e}^{-\sqrt{p / k} x},
$$

where

$$
\begin{aligned}
& A=\frac{\rho}{c}+\mu \varepsilon+\frac{\mu e^{2}}{c}, \quad B=\frac{\mu \varepsilon \rho}{c}, \\
& x_{1}(p)=\frac{k_{1} \theta_{0} p}{k}+\frac{e}{c} k_{2} \theta_{0} \sqrt{p / k}-\mu \varepsilon p^{2} k_{1} \theta_{0}, \\
& x_{2}(p)=\frac{1}{p+\omega}\left[1-\mathrm{e}^{-(p+\omega) \tau}\right] .
\end{aligned}
$$

The solution of Eq. (13) satisfying the regularity condition (Eq. (11c)) is then obtained as

$$
\vec{u}=\alpha \mathrm{e}^{-m_{1} x}+\beta \mathrm{e}^{-m_{2} x}+\frac{x_{1}(p) x_{2}(p)}{x_{3}(p)} \mathrm{e}^{-\sqrt{p / k} x},
$$

where

$$
x_{3}(p)=\frac{\mu \varepsilon \rho}{c} p^{4}-\frac{p^{3}}{k}\left(\frac{\rho}{c}+\mu \varepsilon+\frac{\mu e^{2}}{c}\right)+\frac{p^{2}}{k^{2}},
$$

$\alpha$ and $\beta$ are two constants and $m_{1}, m_{2}$ are the positive roots of the equation $m^{4}-A p^{2} m^{2}+B p^{4}=0$. Now the boundary conditions, Eqs. $(11 \mathrm{a}, \mathrm{c})$, with the aid of Eq. (14) yield

$$
\begin{aligned}
& \alpha m_{1}+\beta m_{2}+T_{0}+\sqrt{p / k} \frac{x_{1}(p) x_{2}(p)}{x_{3}(p)}=0, \\
& \left(\frac{\rho p^{2}}{c m_{1}}-m_{1}\right) \alpha+\left(\frac{\rho p^{2}}{c m_{2}}-m_{2}\right) \beta \\
& +\frac{x_{1}(p) x_{2}(p)}{x_{3}(p)}\left[\frac{\rho}{c} \sqrt{k} p^{3 / 2}-\sqrt{p / k}+k_{1} \theta_{0} \sqrt{k / p} \frac{x_{3}(p)}{x_{1}(p)}\right]=0 .
\end{aligned}
$$

Solving these two equations, we get the values of $\alpha$ and $\beta$. Now, the mechanical displacement on the surface $x$ is

$$
\begin{aligned}
& (\bar{u})_{x=0}=\alpha+\beta+\frac{x_{1}(p) x_{2}(p)}{x_{3}(p)} \\
& =-\left(\frac{c}{\rho p^{2}} \frac{m_{1} m_{2}}{m_{1}+m_{2}}+\frac{1}{m_{1}+m_{2}}\right)\left(T_{0}+\sqrt{p / k} \frac{x_{1} x_{2}}{x_{3}}\right) \\
& -\frac{c}{\rho p^{2}} \frac{m_{1} m_{2}}{m_{1}+m_{2}}\left(\frac{\rho}{c} \sqrt{k} p^{3 / 2}-\sqrt{p / k}+k_{1} \theta_{0} \sqrt{k / p} \frac{x_{3}}{x_{1}}\right) \frac{x_{1} x_{2}}{x_{3}}+\frac{x_{1} x_{2}}{x_{3}},
\end{aligned}
$$


where from the equation $m^{4}-A p^{2} m^{2}+B p^{4}=0$ we get

$$
\frac{m_{1} m_{2}}{m_{1}+m_{2}}=p \lambda_{1} \quad \text { and } \quad \frac{1}{m_{1}+m_{2}}=\frac{\lambda_{2}}{p}
$$

where $\lambda_{1}$ and $\lambda_{2}$ are material constants independent of $p$. Now putting the values of $m_{1} m_{2} /\left(m_{1}+m_{2}\right), 1 /\left(m_{1}+m_{2}\right)$, the expressions for $x_{1}(p), x_{2}(p)$, and $x_{3}(p)$ into Eq. (17) and taking the inverse transform, we get

$$
\begin{aligned}
& u(0, t)=-\left[\left(\frac{c \lambda_{1}}{\rho}+\lambda_{2}\right) T_{0}+\left(\frac{c}{\rho} k_{1} \theta_{0} \lambda_{1} \sqrt{k} \tau-B \tau \mu \varepsilon k_{1} \theta_{0} \lambda_{1} \sqrt{k}\right) \frac{t^{1 / 2}}{\Gamma^{3 / 2}}\right. \\
& +B \tau \mu \varepsilon k_{1} \theta_{0} t-\left(\frac{B \tau \mu \varepsilon k_{1} \theta_{0} \lambda_{2}}{\sqrt{k}}+\frac{\lambda_{1} A \tau \mu \varepsilon k_{1} \theta_{0}}{\sqrt{k}}-\frac{\lambda_{1} B \tau k_{1} \theta_{0}}{\sqrt{k}}\right) \frac{t^{3 / 2}}{\Gamma^{5 / 2}} \\
& +\left(\frac{A \tau}{k} \mu \varepsilon k_{1} \theta_{0}-\frac{B \tau k_{1} \theta_{0}}{k}+\lambda_{1} B \tau \frac{e}{c} k_{2} \theta_{0}\right) \frac{t^{2}}{2}+\left(\frac{\lambda_{1} A \tau k_{1} \theta_{0}}{k^{3 / 2}}\right. \\
& \left.\left.+\frac{\lambda_{1} \tau \mu \varepsilon k_{1} \theta_{0}}{k^{3 / 2}}-\frac{\lambda_{2} A \tau \mu \varepsilon k_{1} \theta_{0}}{k^{1 / 2}}+\frac{\lambda_{1} B \tau k_{1} \theta_{0}}{k^{1 / 2}}-B \tau \frac{e}{c} k_{2} \theta_{0} \frac{1}{\sqrt{k}}\right) \frac{t^{5 / 2}}{\Gamma^{7 / 2}}\right] .
\end{aligned}
$$

For case 2

$$
\begin{aligned}
& \bar{E}_{y}=0, \\
& \bar{\theta}=\frac{\theta_{0}}{p^{2}+\omega^{2}}\left(\omega-p \mathrm{e}^{-p \tau} \sin \omega \tau-\omega \mathrm{e}^{-p \tau} \cos \omega \tau\right), \\
& \bar{T}=T_{0}\left[\frac{1}{p-\omega}-\frac{e^{-(p-\omega) \tau}}{p-\omega}\right], \\
& \bar{\theta}(x, p), \bar{u}(x, p) \rightarrow 0 \text { as } x \rightarrow \infty .
\end{aligned}
$$

Now solving Eq. (9) satisfying the regularity and boundary condition of Eq. (19), we get

$$
\bar{\theta}(x, p)=\frac{\theta_{0}}{p^{2}+\omega^{2}} \mathrm{e}^{-\sqrt{p / k} x}\left(\omega-p \mathrm{e}^{-p \tau} \sin \omega \tau-\omega \mathrm{e}^{-p \tau} \cos \omega \tau\right) .
$$

Substituting the expression for $\bar{\theta}(x, p)$ from Eq. (20) into Eq. (10), we get

$$
\left(D^{4}-A p^{2} D^{2}+B p^{4}\right) \bar{u}=x_{1}(p) x_{4}(p) \mathrm{e}^{-\sqrt{p / k} x},
$$

where

$$
x_{4}=\frac{1}{p^{2}+\omega^{2}}\left(\omega-p \mathrm{e}^{-p \tau} \sin \omega \tau-\omega \mathrm{e}^{-p \tau} \cos \omega \tau\right) .
$$

The solution of Eq. (21) satisfying the regularity condition of Eq. (19c) is then obtained as

$$
\bar{u}=\alpha \mathrm{e}^{-m_{1} x}+\beta \mathrm{e}^{-m_{2} x}+\frac{x_{1}(p) x_{4}(p)}{x_{3}(p)} \mathrm{e}^{-\sqrt{p / k} x},
$$

where $\alpha$ and $\beta$ are two constants which are to be determined from the boundary conditions Eqs. (19a, c) and $m_{1}, m_{2}$ are the positive roots of the equation

$$
m^{4}-A p^{2} m^{2}+B p^{4}=0 \text {. }
$$

Now, the mechanical displacement on the surface $x$ is

$$
(\bar{u})_{x=0}=\alpha+\beta+\frac{x_{1}(p) x_{4}(p)}{x_{3}(p)}
$$




$$
\begin{aligned}
& =\left(\frac{c}{\rho p^{2}} \frac{m_{1} m_{2}}{m_{1}+m_{2}}+\frac{1}{m_{1}+m_{2}}\right)\left[\frac{T_{0}}{p-\omega}-\frac{T_{0} \mathrm{e}^{-(p-\omega) \tau}}{p-\omega}+\sqrt{\frac{p}{k}} \frac{x_{1} x_{4}}{x_{3}}\right] \\
& =-\frac{c}{\rho p^{2}} \frac{m_{1} m_{2}}{m_{1}+m_{2}}\left(\frac{\rho}{c} \sqrt{k} p^{3 / 2}-\sqrt{\frac{p}{k}}+k_{1} \theta_{0} \sqrt{k / p} \frac{x_{3}}{x_{1}}\right) \frac{x_{1} x_{4}}{x_{3}}+x_{1} x_{4} / x_{3},
\end{aligned}
$$

where $m_{1} m_{2} /\left(m_{1}+m_{2}\right)=p \lambda_{1}$ and $1 /\left(m_{1}+m_{2}\right)=\lambda_{2} / p, \lambda_{1}$ and $\lambda_{2}$ are material constants.

Putting the values of $m_{1} m_{2} /\left(m_{1}+m_{2}\right), 1 /\left(m_{1}+m_{2}\right)$, the expressions for $x_{1}(p), x_{4}(p)$ and $x_{3}(p)$ into Eq. (24) and taking the inverse transform, we get

$$
\begin{aligned}
& u(0, t)=-\left[\left(\frac{c \lambda_{1}}{\rho}+\lambda_{2}\right) T_{0} \tau+z_{1} \delta(t)+z_{2} \delta(t-\tau)+z_{3} \frac{t^{-1 / 2}}{\Gamma^{1 / 2}}+z_{4} H(t)\right. \\
& \left.+z_{5} \frac{t^{1 / 2}}{\Gamma^{3 / 2}}+z_{6} t+z_{7} \frac{t^{3 / 2}}{\Gamma^{5 / 2}}\right]
\end{aligned}
$$

where $z_{1}, z_{2}, z_{3}, \ldots z_{7}$ are certain constants.

\section{Discussion}

For numerical computations, the standard values of the material constants for quartz crystals were taken from Cady [3], Kaliski [6], Gibbs [10], Sasaki and Takeuchi [11], Imano and Okuyama [12], etc. while values like $\omega, T_{0}, \theta_{0}, \tau$, and $E$ were chosen suitably to facilitate the numerical computations as follows:

$$
\begin{array}{ll}
\omega=1.57 \mathrm{rad} / \mathrm{s}, & E=300 \mathrm{~V}, \\
T_{0}=1.0 \mathrm{~N} / \mathrm{m}^{2}, & \tau=30 \mathrm{~s}, \quad \theta_{0}=350 \mathrm{~K} .
\end{array}
$$

Equations (19) and (25) have been represented graphically in Figs. 2 and 3 respectively. Both equations consist of a time-independent, as well as a time-de-

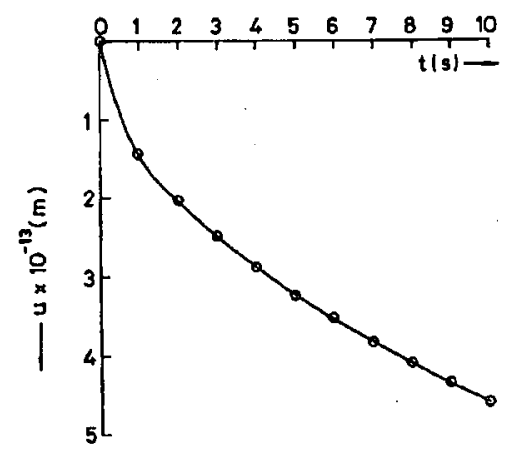

Fig. 2. Variation of mechanical disturbances of piezoquartz along the $x$-direction with time in case 1 .

pendent part. Figure 2 and 3 represent the variation of displacements with time and it is found to be parabolic in both the inputs. The variation of mechanical disturbances along the $x$-direction with time for the above two cases are shown in Tables I and II. 


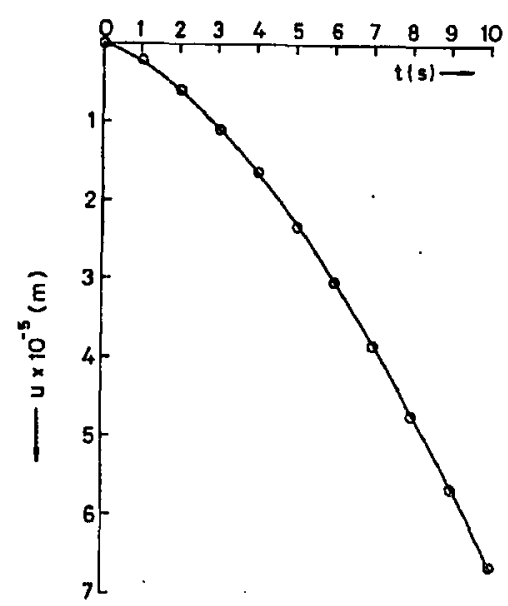

Fig. 3. Variation of mechanical disturbances of piezoquartz along the $x$-direction with time in case 2.

TABLE I

Numerical values of mechanical disturbances along the $x$-direction $(u)$ vs. time $(t)$ for case 1.
TABLE II Numerical values of mechanical disturbances along the $x$-direction $(u)$ vs. time $(t)$ for case 2 .

\begin{tabular}{r|c}
\hline \hline$t[\mathrm{~s}]$ & $u\left[\times 10^{-13} \mathrm{~m}\right]$ \\
\hline 0 & 0.0 \\
1 & -1.46 \\
2 & -2.06 \\
3 & -2.52 \\
4 & -2.92 \\
5 & -3.26 \\
6 & -3.57 \\
7 & -3.86 \\
8 & -4.12 \\
9 & -4.38 \\
10 & -4.61
\end{tabular}

\begin{tabular}{r|c}
\hline \hline$t[\mathrm{~s}]$ & $u\left[\times 10^{-5} \mathrm{~m}\right]$ \\
\hline 0 & 0.0 \\
1 & -0.21 \\
2 & -0.59 \\
3 & -0.09 \\
4 & -1.68 \\
5 & -2.34 \\
6 & -3.08 \\
7 & -3.88 \\
8 & -4.75 \\
9 & -5.67 \\
10 & -6.64
\end{tabular}

\section{References}

[1] J.F. Haskins, L.J. Walsh, J. Acoust. Soc. Am. 29, 729 (1951).

[2] M.C. Mc Quarrie, W.R.J. Buessem, J. Am. Ceram. Soc. 34, 402 (1955).

[3] W.G. Cady, Piezoelectricity, McGraw-Hill, New York 1959. 
[4] R.D. Mindlin, On the Equation of Piezoelectric Crystals: Problems on Continuum Mechanics, Society for Industrial and Applied Mathematics, Philadelphia 1961, p. 282.

[5] M. Redwood, J. Acoust. Soc. Am. 33, 527 (1961).

[6] S. Kaliski, Proc. 'Vibr. Probl. 6, 1 (1965).

[7] M.S. Balabaev, F.N. Ivina, Sov. Phys.-Acoust. (USA) 34, 90 (1988).

[8] L.V. Karlash, Sov. Appl. Mech. (USA) 26, 440 (1990).

[9] J.C. Chen, Appl. Phys. Lett. 60, 132 (1992).

[10] F.D. Gibbs, Ferroelectrics 82, 133 (1988).

[11] Y. Sasaki, Y. Takeuchi, J. Acoust. Soc. Jpn. (E) (Japan) 10, 181 (1989).

[12] K. Imano, D. Okuyama, Trans. Inst. Electron. Inf. Commun. Eng. A (Japan) J74, 797 (1991). 